\title{
FUNCIÓN JUDICIAL Y BIEN COMÚN: LA APORTACIÓN DE LA DEONTOLOGÍA JURÍDICA
}

\author{
CLAUDIO SARTEA
}

\begin{abstract}
Resumen
El artículo intenta profundizar en el concepto de deontología profesional para ponerlo al día y permitirle salir de una crisis de eticidad y sentido de la justicia que parece arrolladora. En la actualidad, se han difundido perspectivas que quitan al trabajo profesional (también al trabajo de los profesionales del derecho, especialmente de los jueces) toda referencia al bien común, reduciéndolo según los casos a simple relación entre sujetos (más o menos económicamente relevante), en la que todo es objeto de negociación, o también a manifestación de un poder público que todo lo controla y reglamenta, acabando paradójicamente por desresponsabilizar a los profesionales. Hay que profundizar en el concepto mismo de trabajo profesional, especialmente, por lo que nos interesa aquí, en la idea y función específica de las profesiones de administración de la justicia, hasta llegar a un modelo de trabajo profesional en el que quien lo ejerce sepa y perciba que está en juego su misma identidad personal: solamente así las reglas cobrarán su sentido y lograrán su fin, que es el de empujar a cada uno a desarrollar acabadamente su papel social y de esta forma a participar por lo que le toca a la realización del bien común.
\end{abstract}

Palabras clave: Jurista - Trabajo - Deontología profesional - Justicia - Bien común.

\begin{abstract}
The article attempts to provide an in-depth study of the concept of professional deontology with the aim of updating it and allowing it to break free from a crisis of ethics and sense of justice that seems overwhelming. At present, a number of perspectives have been disseminated that strip the work of professionals (the work of legal professionals and particularly of judges as well) from any reference to a common good, reducing it, according to each case, to a simple relationship between persons (more or less economically
\end{abstract}

\footnotetext{
* Claudio SARTEA se ha licenciado en Derecho en la Facultad de Derecho de la Universidad de Milán, y ha conseguido el título de Doctor en Filosofía del Derecho en la Facultad de Derecho de la Universidad de Padua. Actualmente es Profesor Titular de Filosofía del Derecho en la Facultad de Derecho de la Universidad de Roma "Tor Vergata" y Profesor de Bioética en la Facultad de Medicina de la Universidad "Campus Bio-medico" de Roma. Desde el año 2012 es profesor visitante de la Universidad Católica Santo Toribio de Mogrovejo de Chiclayo, que lo invita periódicamente para conferencias y clases. Ha publicado numerosos estudios y ensayos, entre cuyos se señalan aquí las monografías: L'emergenza deontologica. Contributo allo studio dei rapporti tra etica, deontologia e diritto, Aracne, Roma, 2007; Deontologia. Filosofia del lavoro professionale, Giappichelli, Torino, 2010; Biodiritto. Fragilità e giustizia, Giappichelli, Torino, 2012; Diritto secolare. Religione e sfera pubblica, oggi, Aracne, Roma, 2012.
} 
relevant), whereby everything is subject to negotiation or to the manifestation of a public power that controls and regulates all things, paradoxically ending up by removing the professional liability. The very concept of professional work must be studied in depth, particularly with respect to the idea and specific function of the professions involved in the administration of justice until we achieve a professional work model, in which person who exercise it, will know and perceive that it is their personal identity which is at stake: only then rules will make sense and achieve their purpose, which is to encourage everyone to conscientiously develop their social role and participate in the attainment of the common good.

Key words: Jurist - Work - Professional Deontology - Justice - Common Good.

\section{Sumario}

1.- Premisas. 2.- Si todo es naturaleza. La abstracción "espontaneísta". 3.Si todo es mercado. El cinismo como cultura. 4.- Si todo es acuerdo. La negociación generalizada. 5.- $\mathrm{Si}$ todo es poder. La negación de la autonomía. 6.- Hacia la libertad. Tomarse en serio la responsabilidad personal.

\section{PREMISAS}

En su obra maestra de filosofía moral, la Ética Nicomaquea, escribe ARISTÓTELES para poner un ejemplo que en su parecer tenía que esclarecer definitivamente un asunto: "Ir delante del juez significa ir delante de la justicia, ya que el juez viene a significar la misma justicia viviente. Y se va en busca del juez como término medio (hasta que unos llaman a los jueces "mediadores"), en la persuasión de que si se alcanza al término medio, se logrará el justo"1.

En el pensamiento clásico, la justicia como exigencia social y como virtud individual, y la concreta actividad de los sujetos encargados institucionalmente de administrarla, eran la misma cosa: hasta el punto de que la persona del juez se convertía en la encarnación de la justicia, en la "justicia viviente". Impresiona la continuidad de esta antigua idea con la moderna afirmación de un conocido jurista italiano, Piero CALAMANDREI: "El juez es el derecho hecho hombre; sólo de este hombre puedo esperar en la vida práctica la tutela que en abstracto me promete la ley; sólo si este hombre sabe pronunciar a mi favor la palabra de la justicia, podré comprender que el derecho no es una sombra vana. Por eso se sitúa en la iustitia, no simplemente en el ius,

\footnotetext{
1 ARIstóteles, Ética Nicomaquea, V, 4, 1132a, 20 (Traducción del autor).
} 
el verdadero fundamentum regnorum; porque si el juez no está despierto, la voz del derecho queda desvaída y lejana, como las inaccesibles voces de los sueños" ${ }^{2}$.

Y del mismo autor son las palabras que poéticamente expresan el simbolismo de la toga: "Amo la toga, no por los adornos dorados que la embellecen, ni por las largas mangas que dan solemnidad al ademán, sino por su uniformidad estilizada, que simbólicamente corrige todas las intemperancias personales y difumina las desigualdades individuales de los hombres bajo el oscuro uniforme de la función. La toga, igual para todos, reduce a quien la viste a ser un defensor del derecho, 'un abogado', como quien se sienta en los sitiales del Tribunal es 'un juez', sin adición de nombres o títulos"3.

Función, institución: ¿de qué estamos hablando, propiamente? Escribe un filósofo español que "una institución es la encarnación social de un bien, es la organización operativa - unas acciones, unos procedimientos, unos actores cuyo funcionamiento es aquello en lo que consiste la misma presencia efectiva de un determinado bien en la sociedad. Una institución es un órgano social que es creado para convertir en una función - en un cometido asignado como propio a un actor particular - la realización de un bien, la existencia de este como contenido estable de la vida social" ${ }^{4}$.

En mi opinión, para entender el sentido profundo de la deontología de las profesiones judiciales es imprescindible colocarse entre esas dos polaridades conceptuales: el juez - y más en general los profesionales del derecho, los así dichos "juristas" - es una persona humana, y al mismo tiempo es una institución. De alguna forma, además de ser sí mismo, es un rol, desarrolla un papel establecido. Interpreta libremente, con sus capacidades y aptitudes, un guión ya escrito: tiene que responder a expectativas colectivas muy concretas y que se han definido y a veces hasta "cristalizado" antes de su llegada al mundo.

Estos conceptos realmente no introducen nada nuevo en la filosofía del trabajo humano: toda actividad supone esta doble vertiente, se mueve entre la dimensión intensamente personal de la libre actuación de un individuo

2 Calamandrei, P. Elogio de los jueces, (1959), trad. esp. De A. Redín, S. Sentís Melendo, C. Finzi, El Foro, Buenos Aires, 1997, p. 70. Agradezco muy especialmente a la Doctora Sandra Brandi de Portorrico haberme facilitado la versión española de este libro italiano: disculpe el lector si no he tenido la misma suerte (y cortesía) con otras obras que en lo sucesivo voy a citar en su versión original o en su traducción italiana.

3 Calamandrei, P., Elogio de los jueces, cit., p. 78.

4 Cruz Prados, A. Filosofía política, EUNSA, Pamplona, 2009, p.38. 
y la dimensión pública y social de tareas y expectativas ${ }^{5}$. Pero en el caso de las profesiones judiciales, y con mucho más motivo en el caso de los jueces, nos encontramos delante de expectativas sociales tan fundamentales como la misma posibilidad de vivir en una sociedad justa, que, ni más ni menos, representa la condición de posibilidad de la coexistencia entre seres humanos.

Las amenazas a la función judicial, y a su correcta ejecución, resultan por lo tanto amenazas muy relevantes al orden social mismo, a la posibilidad de una coexistencia pacífica. Al mismo tiempo, resulta evidente que en nuestra época, así como en las épocas que nos han precedido, el problema de la corrupción de los jueces o más en general de su inidoneidad no ha constituido solo una hipótesis, sino con frecuencia una verdadera llaga social, fuente de tensiones, insatisfacciones e injusticias gravísimas. No veo en esto más que una de las concreciones de lo que Kant afirmaba del hombre en general, diciendo que, si lo imaginamos como una escultura de madera, tenemos que decir que ha sido hecho de una "madera retorcida". En el fondo es, ni más ni menos el franco reconocimiento de nuestra falibilidad, de los límites que tan bien conocemos, y tan a menudo experimentamos, en nuestra condición humana.

En el caso concreto de los jueces, ¿qué soluciones se nos presentan para, no digo solucionar de una vez, sino al menos controlar y limitar este problema tan relevante? Algunos tienen la convicción de que con un adecuado sistema de reglas podremos realizar el paraíso en la tierra: introduciendo una inteligente reforma de la justicia, o medidas eficaces para oponerse a la corrupción y al degrado de la administración de la justicia, o también multiplicando los códigos de ética profesional. Personalmente, opino que la confianza en las reglas no resulte al fin y al cabo suficiente, y que a la actividad de reglamentar, controlar, sancionar, que desde luego resulta imprescindible, no es suficiente, porque tiene que acompañarse a la responsabilidad ética personal de los juristas y su consecuente formación profesional y moral.

Charles TAYLOR, filósofo canadiense, ha hablado en sentido polémico de una verdadera "nomolatría" que en su opinión afligiría a la mentalidad postmoderna como consecuencia de la difusión generalizada del formalismo normativo y de la "ética de la tercera persona". Este autor ha observado que, en vez de mejorar las cosas, la multiplicación de reglas, normas, códigos y leyes se ha convertido muchas veces, paradójicamente, en un nuevo

\footnotetext{
5 Se me permita al respeto remitir a C. SARTEA, Deontologia. Filosofia del lavoro professionale, Giappichelli, Torino, 2010.
} 
mecanismo de desresponsabilización de los individuos ${ }^{6}$. De esta forma, a la vigilancia de la conciencia fundada en conocimientos y evaluaciones objetivas, se sustituye la referencia pasiva a reglas de conducta exterior que solamente piden una adhesión formal y ritual. Clásicamente, la obediencia dependía de la percepción sujetiva del fin que llena de sentido la regla: un bien común que nos embarga a todos y que la autoridad de quien establece la regla nos ayuda a divisar con nuestra libertad e inteligencia. En nuestros días, perdida ya la referencia al fin y desaparecido todo sentido del bien común, nos quedamos con un supino mirar a leyes y códigos donde supuestamente descansa la moralidad pública, con la posible y triste consecuencia que se adormezca la sensibilidad moral de los ciudadanos y de los profesionales.

Resulta por lo tanto imprescindible volver a las raíces de la responsabilidad moral personal, que no estriban en reglas extrínsecas (aún cuando estas resulten necesarias al menos para alumbrar el correcto estilo profesional y conectarlo al fin del bien común), sino en una conciencia profunda, y profundamente formada, de la propia identidad profesional. En este marco me parece importante proponer unas reflexiones acerca de las fuentes culturales de la actual crisis de las profesiones (por lo que nos interesa aquí, de las profesiones judiciales), que por comodidad dividiré en cuatro modelos: modelos no rígidamente separados, y que sobre todo comparten un planteamiento reduccionista de la relación entre el ser humano y su trabajo. Solamente tras aclarar los orígenes de nuestro problema, en mi opinión, será luego posible proponer una "pars construens" en la última, breve parte de mis reflexiones.

\section{SI TODO ES NATURALEZA. LA ABSTRACCIÓN “ESPONTANEÍSTA"}

Una primera genealogía de la crisis en la que nos encontramos hoy, la que me parece más profunda y que por esto merece mayor atención crítica, se puede conectar a la perspectiva antropológica de Jean-Jacques ROUSSEAU. El conocido intelectual de Ginebra se revela como uno de los más convencidos detractores de las instituciones sociales, que en su opinión constituyen, ni más ni menos, la fuente de la corrupción humana - mejor dicho, la causa principal de la salida de los seres humanos de un "estado de naturaleza" considerado como condición inicial de felicidad, de la que el hombre habría decaído por su misma, lamentable iniciativa. En otros términos, cuando aceptamos la vida en común y sus reglas, cuando nos colocamos en la red de relaciones que nos vincula a nuestro origen biológico (la familia) y a nuestro contexto

\footnotetext{
6 TAYLOR, C. L'età secolare, (2007), trad. it. a cura di P. Costa, Feltrinelli, Milano, 2009, p. 887.
} 
social (las amistades, la sociedad), ya "todo se reduce a las apariencias y se vuelve ficticio y simulado"7. Las responsabilidades que dependen del contexto (familiar, profesional, social), se convierten en una negativa y trágica limitación de nuestra originaria libertad.

Me parece evidente cómo en muchas corrientes del pensamiento ético y social moderno se manifieste semejante crítica de todo vínculo a la libre manifestación de la espontaneidad individual (en la que consistiría nuestra más profunda personalidad). En consecuencia, habría que rechazar cualquiera vínculo: por ejemplo, las responsabilidades típicas de nuestro trabajo, de nuestra posición social. ¿Tenemos que aceptar este planteamiento, que claramente elimina toda posibilidad de fundar objetivamente una deontología? Yo creo que nos sería útil reflexionar sobre la relevancia antropológica del contexto y sobre la dignidad propia del rol o papel. Robert SPAEMANN ha escrito, en una de sus obras más sugerentes, que "le persone non sono ruoli, ma esse sono ciò che sono soltanto quando giocano un ruolo, il che significa animando in qualche modo uno stile" . No cabe duda de que ninguno de nosotros puede reducirse (o aceptar verse reducido) a un rol: nuestra personalidad va más allá de todo reduccionismo. Y sin embargo, esta misma personalidad -nuestra siemprese manifiesta y expresa por la mediación de un rol, siempre se relaciona a los demás y vive en su contexto a través de un papel. Como ha observado SPAEMANN, "ho sentito una volta un bambino di cinque anni, alla fine di una seria di giochi di trasformazione, dire a sua madre: 'Io so a che cosa noi adesso possiamo giocare: tu sei la madre, e io sono il bambino'. Dopo i differenti ruoli interpretati $i$ suoi stessi normali rapporti vitali gli apparivano come un ruolo da interpretare" ${ }^{\prime \prime}$.

Cada uno de nosotros - no en abstracto, sino considerado en su existencia concreta, en su realidad efectiva - además que sí mismo en toda su unicidad, es al mismo tiempo un hijo, un hermano, un padre; una mujer o un varón; un joven, un viejo, un hombre maduro; un individuo sano o enfermo; un italiano o un peruano o el ciudadano de otro Estado, de habla determinada y con sus tradiciones culturales, familiares, religiosas; finalmente un estudiante, un profesor, un abogado, un magistrado. En mi persona - y no para cubrir o hasta borrar mi identidad, sino al revés, para afianzarla y manifestarla a los demás - convergen papeles numerosos y coherentes (cuando sean coherentes, es decir en todo individuo no esquizofrénico), que acaban por definirme a mi mismo y presentarme a los demás.

\footnotetext{
7 Rousseau, J.-J. Discurso acerca del origen de la desigualdad entre los hombres (1755).

8 Spaemann, R. Persone. Sulla differenza tra "qualcosa" e "qualcuno", (1998), trad. it. a cura di L. Allodi, Laterza, Roma-Bari, 2005, p. 82.

9 Spaemann, R. Persone, cit., p. 81.
} 
Intentar quitarme esas "máscaras", negando las responsabilidades que implican, equivaldría a abandonar la escena, a salir de la condición humana, a saltar más allá de mi sombra. El mismo RousseAu, en el fondo, "assume un ruolo, che è quello di non interpretare più alcun ruolo. È significativo che egli intenda l'uomo naturale come un ominide privo di parola e di capacità. La dignità umana coincide con l'estraneazione, giacché con la lingua e la divisione del lavoro gli uomini si affrontano interpretando ruoli, anziché essere trasparenti gli uni per gli altri"10.

$\mathrm{Si}$, en cambio, aceptamos nuestro papel, no con resignación sino con la plena convicción de que se trata de nuestro lugar propio en la vida y en la sociedad, estamos en condiciones de contestar afirmativamente a la pregunta ética por excelencia: la que Dios dirigió a Adán en el Jardín de Edén, tras la primera, dramática asunción de responsabilidad moral de los hombres: “¿Donde estás?"11. En aquel crucial momento, el hombre no se encontraba en el lugar donde Dios lo buscaba, es decir en el lugar donde tenía que estar porque allí Dios mismo lo había colocado. Como nos explica Paul Ricoeur, la pregunta acerca del "dónde" es la pregunta moral por excelencia: "Il mantenersi [permanecer fielmente en el propio lugar] per la persona è la tale maniera di comportarsi grazie alla quale l'altro può contare su di lei. Poiché qualcuno conta su di me, io sono in grado di render conto delle mie azioni davanti ad un altro. Il termine di responsabilità raccoglie le due significazioni: contare su... essere in grado di render conto di... Essa le raccoglie, aggiungendovi l'idea di una risposta alla questione: 'Dove sei?', posta dall'altro che mi reclama. Tale risposta è: 'Eccomi!'. Risposta che dice il mantenersi"12.

\section{SI TODO ES MERCADO. EL CINISMO COMO CULTURA}

Un segundo reto que la cultura moderna - así como la actual organización del trabajo - dirige a la deontología jurídica, consiste en mi opinión, en la reducción de las profesiones al mercado. No cabe duda de que uno de los significados y de las funciones típicas de la actividad laboral es permitir a quien lo ejerce sustentarse a sí y a la propia familia, y en esto no veo ninguna dificultad y riesgo: sin embargo, lo que aquí estoy considerando es el peligro, más actual que nunca en tiempos de economicismo desencadenado, que el trabajo quede reducido a su valor económico.

\footnotetext{
10 SpaemanN, R., Persone, cit., p. 84. Más detenidamente sobre el pensamiento de Rousseau R. SPAEMANN, Rousseau. Cittadino senza patria. Dalla 'polis' alla natura, (2008), trad. it. a cura di L. Allodi, Ares, Milano, 2009. 11 Génesis, III, 9.

12 Ricoeur, P.Sé come un altro, (1990), trad. it. a cura di D. Iannotta, Jaca Book, Milano, 1993, p. 259.
} 
Radicalizar esta lectura del trabajo implica de hecho o de principio quitarle toda relevancia moral: en cierto sentido supone pensarlo como una necesidad de sobrevivir en la que nuestra libertad prácticamente no se encuentra afectada, y por consecuente no aparece nuestra responsabilidad moral. Si todo trabajo profesional sólo es cuestión de dinero, de ganarse la vida, ya no queda espacio para compromisos éticos: "quien más gane mejor trabaja", sin preocupaciones de otro tipo. Lo cual, entre otras tristes consecuencias, acaba por legitimar toda corrupción bajo el sello de la necesidad o de la insignificancia moral de la profesión.

"Perché preoccuparsi del fatto che stiamo andando verso un tipo di società in cui tutto è in vendita? Per due ragioni: una riguarda la disuguaglianza; l'altra la corruzione. [...] In una società in cui tutto è in vendita, la vita è più difficile per chi dispone di mezzi modesti. Più il denaro può comprare, più la ricchezza (o la sua mancanza) conta. [...] La seconda ragione per cui dovremmo esitare a mettere tutto in vendita è più complessa da spiegare. Non si guarda la disuguaglianza e l'equità ma gli effetti corrosivi dei mercati. Assegnare un prezzo alle cose buone può corromperle. Questo perché $i$ mercati non solo distribuiscono beni: essi esprimono e promuovono anche determinati atteggiamenti nei confronti dei beni oggetto di scambio"13.

Existen, en otras palabras, cosas que no siguen simplemente la lógica del mercado: en mi opinión, el trabajo humano está entre estas "cosas", mejor dicho, ya que no es simple cosa sino actividad libre de seres racionales y relacionales, no puede no estar entre semejantes "cosas", por naturaleza propia. "El trabajo no es una mercancía", fue afirmado con fuerza en la Declaración de Filadelfia por la Organización Internacional del Trabajo en 19444" lo cual desde luego no implicaba la negación de su relevancia económica, sino su pertenencia a un nivel superior del de las mercancías, del simple mecanismo de los intercambios económicos. Mucho más que un intercambio de cosas y monedas está en juego cuando el hombre trabaja: y esto confiere a sus esfuerzos, también a los más manuales y musculares, una dignidad superior, y fundamenta una responsabilidad ética verdadera.

\section{SI TODO ES ACUERDO. LA NEGOCIACIÓN GENERALIZADA}

Más refinada, y sin embargo más ambigua y peligrosa, resulta una tercera forma de mirar al trabajo: reduciéndolo al acuerdo entre dos voluntades

\footnotetext{
${ }^{13}$ SANDEL, M.J. Quel che il denaro non può comprare. I limiti morali del mercato, (2012), trad. it. a cura di C. Del Bò, Feltrinelli, Milano, 2013, p. 16.

$14 \mathrm{Al}$ tema está dedicado el interesante estudio de L. GALLINO, Il lavoro non è una merce. Contro la flessibilità, Laterza, Roma-Bari, 2007.
} 
arbitrarias. Aún sin la mediación del dinero, hay quienes conciben al trabajo como una negociación permanente y total de bienes e intereses, entre el sujeto genérico que necesita algo, y el profesional que, por su preparación teórica y sus habilidades prácticas, se presenta como capaz de satisfacer sus requerimientos. Resulta evidente que parecida perspectiva borra de la relación profesional toda dimensión ética vinculante: se trata de una simple relación de debe y haber que, si no es de violencia y prevaricación de un sujeto hacia otro, se reduce a una superposición accidental de intereses materiales.

En parecida perspectiva, se pierde de vista el bien objetivo que justifica en el plan teorético y explica en el plan histórico la existencia de toda profesión. Pensamos por ejemplo en los médicos: si no es por su referencia al bien objetivo de la salud, no tiene sentido el ejercicio de un poder tan incisivo en la intimidad corporal de sus iguales. Cuando se afirma que el fundamento de la actividad médica es el "informed consent" del paciente, se olvida que por este camino se puede legitimar cualquiera intervención, desde las más absurdas operaciones de cirugía estética hasta el "suicidio asistido". De hecho, la vertiente que radicaliza la autodeterminación del hombre hasta superar todo límite ético en la actividad de investigación y experimentación científica, y en la actuación clínica, rechaza toda vinculación moral y toda posible finalización objetiva de la profesión médica.

Dudo que este planteamiento mejore la calidad de los servicios profesionales, y me permito sugerir profundizar en la idea de una finalidad intrínseca de las profesiones (su télos objetivo específico como justificación y también como límite operativo: en el caso de la medicina, la restitución de la salud o la paliación del sufrimiento), a la luz del cual habrá en cada caso que valorar la oportunidad y la legitimidad de toda intervención y actuación, más allá del consentimiento informado y del intercambio de voluntades entre los protagonistas. El diálogo terapéutico sin duda es esencial: pero para dialogar hay - primera condición - que hablar el mismo idioma, y - segunda - que aceptar que existen significados (y finalidades) objetivos, que constituyen al mismo tiempo el sentido y el horizonte del diálogo mismo.

Del mismo modo que no puede existir profesionalidad médica sin referencia al bien de la salud, no puede existir administración de la justicia sin referencia a la justicia misma, así como resulta inconcebible la actividad política sin referencia al bien común ${ }^{15}$. Dicho de otra forma, profundizar en la teleología

15 Como escribe A. CRUz PRADOS, Filosofía política, cit., p. 48: "La sociabilidad humana implica que el hombre alcanza su actualización o perfección en sociedad. El bien humano es un bien común. Los mejores y más 
de una profesión, convencerse y formarse en su sentido propio y en las finalidades que la caracterizan y justifican, supone garantizar a la deontología profesional.

\section{SI TODO ES PODER. LA NEGACIÓN DE LA AUTONOMÍA}

Existe desde luego, el peligro opuesto: en obsequio a una concepción anacronística, diría yo "napoleónica" de la vida civil, o simplemente por reacción a los riesgos o a los excesos de la anterior perspectiva individualista, se puede difundir una extremada "publicización", una radical estatalización de las profesiones judiciales (no solamente la del juez, sino también la del abogado), que con el objetivo de garantizar cuanto más posible a los ciudadanos y evitar dichos abusos, de hecho coloquen bajo el completo control del poder estatal la actividad profesional inherente a la administración de la justicia (y no me refiero, claro está, a la reglas procesales o de organización de la magistradura, sino a su ética y deontología propia). No podemos subestimar los riesgos de esta postura: si el juez es simplemente "bouche des lois", como decían los juristas de la Ilustración, la jurisprudencia se convierte en actividad automática y rígida, perdiendo sus características de sabiduría práctica. Si la conducta de los abogados cae bajo la legislación del Estado (es lo que pasa en Francia, por ejemplo, donde los códigos profesionales son promulgados por el ministerio competente por materia), se quita autonomía (es decir, responsabilidad) a los profesionales del derecho, que en cambio son los mejores artífices de su propia disciplina deontológica y desde siempre han elaborado por sí mismos sus reglas profesionales.

Quien conoce algo de la historia reciente del pensamiento jurídico teórico, reconoce fácilmente en lo que estoy diciendo uno de los efectos del iuspositivismo voluntarista: en esta perspectiva, "el intérpretes de la ley sólo tendría que recoger la voluntad del legislador, sin cuestionar acerca de su razonabilidad. Quien tiene que obedecer [el ciudadano, así como el profesional de la justicia] tiene que hacer lo que quiso el legislador, con independencia del hecho que esto resulte bueno o malo"16. De esta forma se debilita no solamente el papel del profesional del derecho, sino el sistema mismo de la administración de la justicia. Aun cuando se pretende garantizar, en cambio, se le acaba por suprimir del todo: una vez más la "nomolatria" es una tentación tan fuerte al comienzo como decepcionante al final.

propios bienes del hombre son bienes comunes, bienes que solo se alcanzan y poseen en común, es decir, son bienes cuya realización consiste en el perfeccionamiento de las comunidades a las que el hombre pertenece".

16 Hervada J. ¿Qué es el derecho? La moderna respuesta del realismo jurídico, Pamplona, 2008, p. 84. 


\section{HACIA LA LIBERTAD. TOMARSE EN SERIO LA RESPONSABILIDAD PERSONAL}

¿Quién es un jurista? No solamente un "hombre de ley": es más, es un "hombre de derecho"17. O, si se prefiere, el hombre del derecho: quien lo hace vivir entre los hombres, para pacificar sus relaciones, hacerlas justas y salvarlas de la violencia y de la irracionalidad. La justicia, como característica imprescindible en toda sociedad humana en la que se pueda vivir, necesita esos obreros humildes y tenaces que somos los juristas. Nótese que es preferible hablar del jurista como hombre del derecho, y no simplemente de las leyes, ya que por lo que hemos hasta ahora considerado no es nada cierto que el derecho solo consista en las normas contenidas en las leyes. Las leyes constituyen, si están bien hechas, la pauta en la que el jurista tiene que trabajar para sacar las reglas del derecho, para decir lo que es justo en el caso concreto.

La mediación racional del juez es por lo tanto imprescindible: y no puede de ninguna manera resultar contagiada, condicionada por elementos que no tengan directamente que ver con la justicia del caso concreto. Toda distorsión de esta perspectiva acaba por ser origen de injusticias: pero nótese que en su raíz consiste en una falta deontológica, en un defecto de profesionalidad.

En su delicada y difícil tarea profesional de decir lo que es justo, de decir el derecho a partir de las leyes, el juez no está solo: lo acompañan y ayudan (así: ayudan) el abogado y el fiscal, y todos los demás profesionales de la justicia. Cada uno tiene que hacer con escrúpulo profesional su tarea, desde el comienzo hasta el final: únicamente así el resultado podrá aspirar a realizar la justicia a la que mira. Y ¿cómo se desarrolla esta colaboración? Precisamente en el proceso, o en relación al proceso. Por esto el proceso tiene tanta trascendencia, necesita reglas claras y sobre todo supone principios tan inflexibles como el de la imparcialidad del juez ${ }^{18}$, del derecho de defensa, del contradictorio $^{19}$, de la publicidad de $\operatorname{los}_{\text {actos }}{ }^{20}$, de la pluralidad de instancias, etc. Cada uno de estos principios remite a bienes esenciales en las relaciones jurídicas, es decir, fundamentales para la vida social y el bien común.

\footnotetext{
17 HeRvadA J., ¿Qué es el derecho? La moderna respuesta del realismo jurídico, cit., p. 17. En argumento véase también M. LA TORRE, Il giudice, l'avvocato e il concetto di diritto, Rubbettino, Soveria Mannelli, 2002.

18 TRujlllo, I. Imparzialità, Giappichelli, Torino, 2003. Más reciente de la misma autora el estudio Etica delle professioni legali, Il Mulino, Bologna, 2013.

19 Cfr. Moro, P. L'etica del contraddittorio. Il principio costitutivo della deontologia forense, in AA.VV., Audiatur et altera pars. Il contraddittorio tra principio e regola, a cura di M. Manzin e F. Puppo, Giuffrè, Milano, 2008, p. 276.

20 MACIOCE, F. La lealtà. Una filosofia del comportamento processuale, Giappichelli, Torino, 2005.
} 
Garantizar que cada uno de los protagonistas del proceso actúe con intensa fidelidad a este cometido es imprescindible para alcanzar esos objetivos, para realizar esos bienes: en este sentido, toda deontología se reduce a un compromiso de fidelidad a la identidad profesional y, por consecuente, al papel que cada uno tiene que desarrollar. Esto explica la importancia de la ética de las profesiones judiciales: que en definitiva a eso principalmente sirven, a garantizar que el proceso sea lo que tiene que ser y no un ritual del que ha sido borrada toda sustancia social, toda finalidad propia, toda su configuración institucional propia de lugar de realización pública de la justicia.

"Il diritto è essenzialmente 'processualità'. Esso vale come valore perché fa valere. E questo suo 'far valere' implica, per l'appunto, la possibilità della lite, del processo e del giudizio"21. El conjunto de las reglas que dirigen el proceso, entonces, llega a cobrar una importancia decisiva: y no me refiero solo a las reglas legales, al derecho procesal en sentido estricto, sino también a las reglas que afectan a la conducta de los protagonistas del proceso mismo. Aquí la deontología profesional muestra su papel central, exactamente en el lugar donde "verdad y libertad coinciden", como escribe Giuseppe CAPOGRASSI: "Qui appare perché $i$ vecchi [los antiguos] vedevano come elemento del giudicare, il 'rectitudinis zelus' cioè l'amor justitiae, l'apertura alla verità, la volontà di realizzare quello che è giusto. Qui si potrebbe dire libertà e verità coincidono. C'era in quella vecchia scienza, con il disperato sogno di sopprimere tutto il soggettivo, di fare arrivare tutto all'obiettività, c'era questa intuizione profonda, che quando tutto è stato adempiuto per arrivare alla verità, cioè quando nella ricerca della verità non si è seguito altro che la linea sicura della logica obiettiva dell'azione e della legge e nell'ordine in cui la ricerca deve svolgersi, la decisione suprema dell'animo del giudice, aperto alla verità perché ha seguito quella pura logica, porta spontaneamente alla verità: la via della ricerca ne garentisce il risultato; la purezza dei mezzi garentisce la verità del fine"22.

Hablar de pureza ya es hablar de virtud: una virtud en este caso dianoética, diría Aristóteles, es decir la virtud de un intelecto que conoce y re-conoce, sin prejuicios. Luchar contra los prejuicios, abandonar posturas ideológicas, no ceder a la tentación de una alteración anómala (por dinero, por simpatías, por lo que sea) de los equilibrios y de los papeles procesales: todo esto hace parte de la tarea judicial (de la salvación del juicio) y no se puede abandonar sin grave menoscabo de toda esperanza de justicia concreta.

\footnotetext{
${ }^{21}$ OPOCHER, E. Lezioni di filosofia del diritto, CEDAM, Padova, 1993, p. 307.

22 G. CAPOGRASSI, Giudizio processo scienza verità, en "Rivista di diritto processuale", 1950/I, pp. 1-22, ahora también en ID., Opere, V, Milano, 1959, pp. 53 y ss.
} 
Pero, antes que vínculos, esos deberes morales y deontológicos resultan una vez más un ejercicio de libertad: la manera concreta de ser lo que elegí ser profesionalmente, juez, abogado, jurista; en otros términos, la forma efectiva de encarnar mi rol social, de interpretar un papel que no es poder, sino servicio, mi manera concreta de contribuir al bien común de la sociedad en la que vivo y a la que debo mi existencia.

Es tan elevado y noble este cometido, es tan delicada y trascendente la función judicial, que podemos añadir a lo que de ella hemos dicho al comienzo algo que todavía ha quedado en el fondo. Es decir que se trata de una tarea "divina" 23: de uno entre los más ambiciosos intentos de los hombres de introducir la justicia en el mundo, de participar en la incansable tarea de salvar al mundo - que es tarea de Dios, participada a los hombres. Muy grave resultaría, con mayor razón en esta perspectiva, ensuciar la dignidad de esta función descuidando su deontología y obedeciendo a lógicas que nada tienen que ver con la justicia como bien común.

23 Cfr. S. SATTA, Il mistero del processo, Adelphi, Milano, 1994; F. D'Agostino, Corso breve di filosofia del diritto, Giappichelli, Torino, 2011, p. 128. 


\section{REFERENCIAS BIBLIOGRÁFICAS}

ARISTÓTELES, Ética Nicomaquea.

Calamandrei, Piero, Elogio de los jueces, (1959), trad. esp. De A. Redín, S. Sentís Melendo, C. Finzi, El Foro, Buenos Aires, 1997.

CAPOGRASSI, Giuseppe, Giudizio processo scienza verità, en ID., Opere, V, Milano, 1959.

Cruz Prados, Alfredo, Filosofía política, EUNSA, Pamplona, 2009.

D' Agostino, Francesco, Corso breve di filosofia del diritto, Giappichelli, Torino, 2011.

GALLINo, Luciano, Il lavoro non è una merce. Contro la flessibilità, Laterza, RomaBari, 2007.

HERVADA, Javier, ¿Qué es el derecho? La moderna respuesta del realismo jurídico, Pamplona, 2008.

MACIOCE, Fabio, La lealtà. Una filosofia del comportamento processuale, Giappichelli, Torino, 2005.

Moro, Paolo, L'etica del contraddittorio. Il principio costitutivo della deontologia forense, in AA.VV., Audiatur et altera pars. Il contraddittorio tra principio e regola, a cura di M. Manzin e F. Puppo, Giuffrè, Milano, 2008.

OPOCHER, Enrico, Lezioni di filosofia del diritto, CEDAM, Padova, 1993.

Ricoeur, Paul, Sé come un altro, (1990), trad. it. a cura di D. Iannotta, Jaca Book, Milano, 1993.

ROUSSEAU, Jean-Jacques, Discurso acerca del origen de la desigualdad entre los hombres, 1755.

SANDEL, Michael J., Quel che il denaro non può comprare. I limiti morali del mercato, (2012), trad. it. a cura di C. Del Bò, Feltrinelli, Milano, 2013.

SARTEA, Deontologia. Filosofia del lavoro professionale, Giappichelli, Torino, 2010. 
Claudio Sartea - Función judicial y bien común: la aportación de la Deontología Jurídica

SATTA, Salvatore, Il mistero del processo, Adelphi, Milano, 1994.

SPAEMANN, Robert, Persone. Sulla differenza tra "qualcosa" e "qualcuno", (1998), trad. it. a cura di L. Allodi, Laterza, Roma-Bari, 2005.

Spaemann, Robert, Rousseau. Cittadino senza patria. Dalla 'polis' alla natura, (2008), trad. it. a cura di L. Allodi, Ares, Milano, 2009.

TAYLOR, Charles, L'età secolare, (2007), trad. it. a cura di P. Costa, Feltrinelli, Milano, 2009.

TRUJILLO, Isabel, Imparzialità, Giappichelli, Torino, 2003.

TRujILLO, Isabel, Etica delle professioni legali, Il Mulino, Bologna, 2013. 